\title{
Point-of-Care Ultrasound Education for Pediatric Residents in the Pediatric Intensive Care Unit
}

\author{
Ryan Good, MD*, Jonathan Orsborn, MD, Timothy Stidham, MD \\ *Corresponding author: ryanjgood@gmail.com
}

\section{Abstract}

Introduction: Point-of-care ultrasound (POCUS) is ultrasound performed by the provider at the patient's bedside to answer a specific clinical question. No guidelines exist for teaching POCUS to pediatric residents, and there are currently no pediatric-specific POCUS resources on MedEdPORTAL. To fill this gap, we designed an educational resource to introduce pediatric residents to POCUS during their pediatric intensive care unit (PICU) rotation. Methods: Our POCUS curriculum included content on ultrasound basics, lung ultrasound, and focused cardiac ultrasound. Residents completed a precourse knowledge test at the start of the PICU rotation. Self-study modules were provided to the residents for independent review. During small group, residents performed ultrasound scanning on subjects with normal anatomy. Residents also participated in weekly POCUS rounds to perform supervised ultrasound scanning on PICU patients with known abnormal ultrasound findings. After completion of the PICU rotation, residents competed a postcourse knowledge test and survey. Knowledge test scores were compared to a historical cohort of residents who had completed the PICU rotation but not the POCUS curriculum. Results: Six residents completed the curriculum, and all completed the postcourse knowledge test with significant improvement in test scores compared to a historical cohort. Residents reported increased knowledge of POCUS indications and comfort performing POCUS. All residents rated the smallgroup sessions and POCUS rounds highly. Discussion: Pediatric residents have little POCUS training and perform poorly on POCUS knowledge testing. A basic POCUS curriculum can be instituted during the PICU rotation and improve resident knowledge and comfort with POCUS.

Keywords

Ultrasound, Pediatrics

\section{Educational Objectives}

By the end of this activity, learners will be able to:

1. Explain the basic physics and function of ultrasound technology.

2. List the potential applications of point-of-care ultrasound (POCUS) in pediatrics.

3. Demonstrate the technical skills necessary to identify a pleural or pericardial effusion using POCUS.

Introduction

Point-of-care ultrasound (POCUS) is ultrasound performed by the provider at the patient's bedside to answer a specific clinical question. It is now incorporated into numerous medical specialties for a variety of applications. ${ }^{1}$ Reflecting this growing utilization, ultrasound is now a key component of the curriculum in many medical schools. ${ }^{2}$ Within graduate medical education, emergency medicine has pioneered utilization of and education in POCUS for the past 3 decades. In 2009, the American College of Emergency Physicians published a POCUS curriculum for emergency medicine providers that includes both didactic and experiential learning prior to demonstration of proficiency. ${ }^{3}$ Other specialties, including pediatric emergency medicine and adult critical care medicine, have followed this educational model to develop specialty-specific POCUS curricula. ${ }^{4,5}$ MedEdPORTAL currently features examples of both didactic and experiential POCUS content for providers in adult critical care medicine. ${ }^{6,7}$

Citation: Good R, Orsborn J, Stidham T. Point-of-care ultrasound education for pediatric residents in the pediatric intensive care unit. MedEdPORTAL. 2018;14:10683. https://doi.org/10.15766/mep_23748265.10683

Copyright: $\odot 2018$ Good et al. This is an open-access publication distributed under the terms of the Creative Commons Attribution-NonCommercialShare Alike license.

Appendices

A. Precourse Knowledge Test docx

B. US Basics Module.pptx

C. Lung US Module.pptx

D. Focused Cardiac US Module.pptx

E. POCUS Rounds Overview docx

F. Postcourse Knowledge Test.docx

G. Precourse Knowledge Test Key.docx

H. Postcourse Knowledge Test Key.docx

All appendices are peer reviewed as integral parts of the Original Publication. 
Due to its lack of ionizing radiation, as well as its availability and its ability to be performed without sedation, ultrasound is an ideal imaging modality for children, and numerous pediatric-specific POCUS applications are clinically relevant. ${ }^{8}$ Despite the potential benefits of POCUS for children, no guidelines exist for training for pediatric residents in POCUS, and few pediatric-specific POCUS training tools exist. We conducted a local POCUS needs assessment among the pediatric residents at the University of Colorado and also demonstrated the need for POCUS training resources. Most of the residents reported no previous POCUS training, and lack of training was identified as the main barrier to POCUS utilization. Precourse POCUS knowledge test scores were poor and showed no improvement between years, indicating lack of POCUS knowledge acquisition as part of pediatric residency training.

Patients in the pediatric intensive care unit (PICU) frequently have abnormal findings that can be visualized with ultrasound. In addition, POCUS use is common in the PICU, and thus, ultrasound machines are routinely available for both clinical and teaching purposes. ${ }^{9}$ The combination of patients, equipment, and expertise make the PICU an ideal environment for the experiential learning necessary for POCUS education. We decided to institute a curriculum with both didactic and experiential components to introduce pediatric residents to POCUS during their PICU rotation.

\section{Methods}

The curriculum was designed for pediatric residents at the University of Colorado to complete during the 1month PICU rotation. Residents complete the PICU rotation during the second postgraduate year and have no prior PICU experience. The PICU at Children's Hospital of Colorado is a mixed medical/surgical unit with a maximum capacity of 40 beds. During the PICU rotation, pediatric residents follow up to five patients per day with supervision from a PICU fellow and attending physician. Residents also admit patients during overnight call shifts that occur once every 6 days. The curriculum content was created by authors who are attending physicians in pediatric critical care medicine and pediatric emergency medicine, with specific training in POCUS.

Prior to participation in the curriculum, residents completed a precourse knowledge test (Appendix A, answer key in Appendix G) to measure their baseline knowledge about POCUS. The test was made up of multiple-choice and true-false questions. It included ultrasound images and took the residents approximately 15 minutes to complete. Residents were instructed not to utilize any resources when completing the test but were not supervised. We utilized a hard copy of the test, but an online version would allow for incorporation of ultrasound videos in addition to still images. After completion of the precourse knowledge test, residents were provided with three learning modules for independent study (Appendices B-D). The modules focused on three main POCUS content areas: (1) ultrasound basics, (2) lung ultrasound, and (3) focused cardiac ultrasound. We sent the modules to the residents via email, but they could also be posted to an online learning platform. We anticipated that it would take the residents 15 to 20 minutes to review the modules independently. We utilized this asynchronous educational approach to allow the residents to have more hands-on time with the ultrasound machine and instructors during the subsequent small-group sessions. Since POCUS is a technical skill, we felt instructor time was more efficiently spent during hands-on sessions rather than in didactic sessions. Alternatively, an instructor could present the material in the modules in a lecture format.

In the first 2 weeks of the PICU rotation, residents participated in a pair of 60-minute, small-group, handson sessions. Six residents participated in each session. The first session covered ultrasound basics and lung ultrasound (Appendices B \& C), while the second session covered focused cardiac ultrasound (Appendix D). Residents were expected to have completed the modules prior to each session. For the first 10 minutes of the session, instructors reviewed the content of the modules and answered resident questions. For the first session, important content to review prior to ultrasound scanning included (1) ultrasound machine basics such as power button location, gain, and depth buttons; (2) linear and 
curvilinear probes and how to change probes in the ultrasound machine; and (3) image acquisition locations for pneumothorax and pleural effusion. For the second hands-on session, less time was spent on reviewing module content since the residents had already been exposed to the ultrasound machine during the first session. During this session, the residents listed the components of a focused cardiac ultrasound exam and identified the cardiac probe prior to ultrasound scanning. For the majority of the hands-on session, residents practiced POCUS technique with the bedside ultrasound machine available in the PICU. There are several options for examples of normal anatomy, including (1) patients with normal anatomy in the PICU, (2) volunteers from ultrasound interest groups at local medical schools, and (3) professional patients (if resources allow). Instructors utilized example images and videos from the modules to compare to the real-time ultrasound images captured by the residents when scanning. During our sessions, instructors had only one ultrasound machine available, so residents took turns practicing ultrasound technique. In order to engage the other residents during the session, instructors asked the residents who were not scanning to identify anatomic structures on the ultrasound display using the modules as a reference.

During the last 2 weeks of the PICU rotation, residents participated in weekly, 1-hour POCUS rounds led by us. To standardize the approach, we created a document that describes the procedure of POCUS rounds (Appendix E). The instructor first identified two to four patients admitted to the PICU with known abnormal findings that could be visualized using ultrasound. Next, the instructor would confirm the safety and appropriateness of using a patient as a model for POCUS rounds with the medical team, including the bedside nurse and attending physician. Finally, the instructor would approach the patient and family to introduce the concept of POCUS rounds and obtain permission.

The process of patient selection takes approximately 1 hour and should be completed shortly before POCUS rounds are scheduled to begin. After patient selection was completed, the instructor would collect the ultrasound machine and a mobile computer and meet the group of six residents at the charge nurse station. The entire group then moved to the bedside of the first patient, and the resident following that patient would provide a short clinical summary of the patient's diagnosis and PICU course. Any relevant imaging would then be reviewed on the mobile computer. The instructor identified a learning objective for ultrasound scanning on the patient based on the diagnosis and known abnormal findings. Examples of learning objectives from POCUS rounds included identifying a pleural effusion with ultrasound and identifying a pneumothorax with ultrasound. The group then entered the patient room, and residents took turns ultrasound scanning on the patient to identify the abnormal finding. A checklist could be utilized to document that the residents had completed important steps in a POCUS examination, including patient position, probe selection, probe position, image acquisition, and image interpretation. After each resident was able to identify the abnormal finding with ultrasound, the group moved to the next patient room and repeated the procedure. POCUS rounds also provided an opportunity for residents to identify normal ultrasound findings in some patients, building on their experience in the small-group sessions earlier in the rotation.

To assess our POCUS curriculum, residents completed a postcourse knowledge test and survey (Appendix F, answer key in Appendix H). The postcourse knowledge test had similar structure and content to the precourse knowledge test. In the survey, residents reported comfort with their ability to perform POCUS. They also rated the components of the POCUS curriculum. Resident knowledge test scores before and after completing the POCUS curriculum were compared. We also compared resident knowledge test scores after completing the POCUS curriculum to a historical cohort of residents who had completed the PICU rotation but not the POCUS curriculum. Given the small sample size, all data were expressed as median with the interquartile range.

\section{Results}

After completion of the curriculum, all six residents completed the postcourse knowledge test, with most (five out of six) demonstrating an improvement in score (Figure 1). 


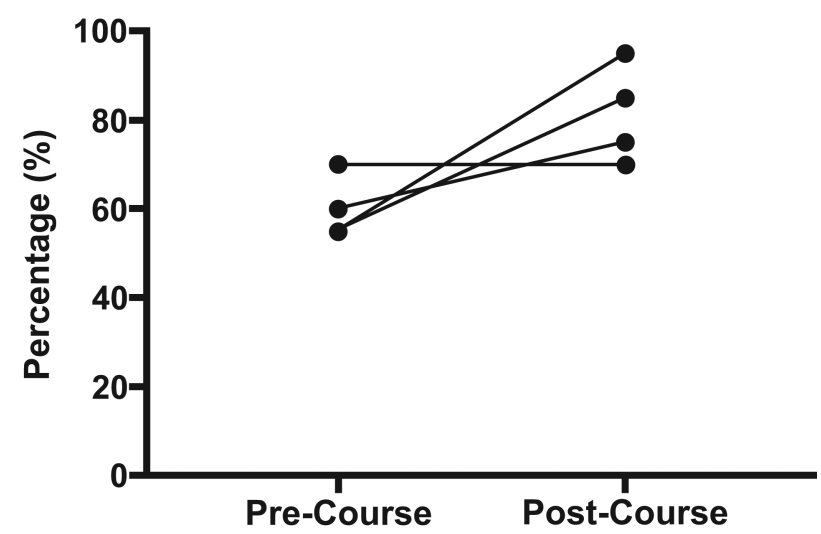

Figure 1. Precourse $(M d n=55 \%$, interquartile range: $55.0 \%-62.5 \%)$ versus postcourse $(M d n=90 \%$, interquartile range: $73.8 \%-95.0 \%)$ knowledge test scores for cohort of residents who completed the curriculum $(N=6)$.

Residents who completed the curriculum had significantly higher scores on the knowledge test than a historical cohort of pediatric residents who had completed the PICU rotation but not the curriculum (Figure 2).

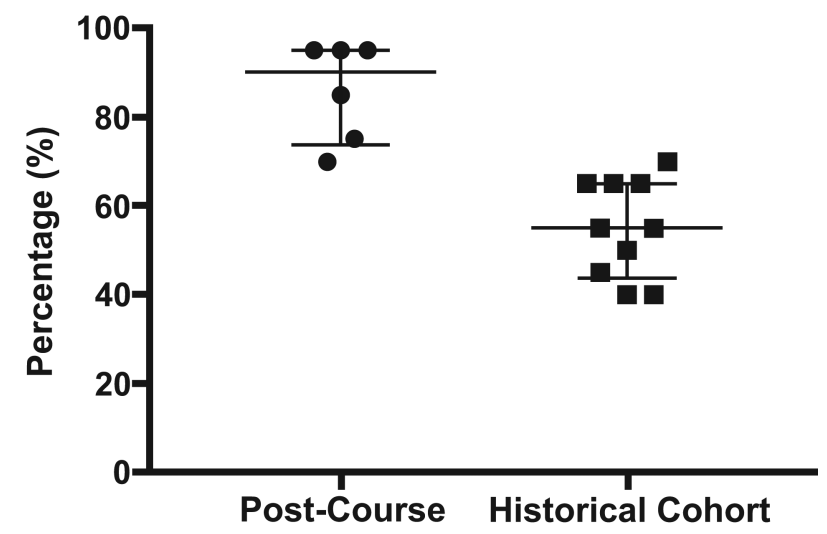

Figure 2. Knowledge test scores of residents who completed the curriculum $(N=6)$ versus a historical cohort of residents who completed the PICU rotation but not the curriculum $(N=10)$. Data are expressed as median and interquartile range; $p<.005$ by Mann-Whitney test.

Residents who completed the curriculum reported increased knowledge of POCUS indications and increased comfort with performing POCUS. Most of the residents who participated in the curriculum $(66.6 \%, N=6)$ anticipated that they would use POCUS in their clinical practice. Residents rated both the small-group hands-on session and POCUS rounds highly.

\section{Discussion}

We developed a novel teaching resource to introduce pediatric residents to selected POCUS applications during the PICU rotation. As our needs assessment demonstrated, our resource fills an important gap in pediatric resident education as residents reported little POCUS training and a lack of comfort in performing POCUS. They also scored poorly on a POCUS knowledge test. We designed a curriculum that incorporated both didactic and experiential learning experiences and increased resident POCUS knowledge compared to a historical cohort of residents. In addition, after completing the PICU rotation and our curriculum, residents reported increased comfort with performing POCUS. 
Our resource has several limitations. We only performed a postcourse knowledge test in a small pilot group of residents and did not perform follow-up assessments at later time points to assess for retention of POCUS skills and knowledge. We also did not have a formal method for assessing resident technical skill with POCUS and instead relied on resident report of comfort. While our curriculum was designed for pediatric residents in the PICU, the modules we created could be used by a number of different learners and in other clinical settings. The curriculum was designed as an introduction to POCUS because pediatric residents receive no formal POCUS training. Thus, the modules could be utilized by other learners with no previous POCUS experience. The applications of lung ultrasound and focused cardiac ultrasound are not specific to pediatric patients and could be utilized by learners in other medical specialties, including internal medicine, emergency medicine, and surgery. Since POCUS is a technical skill, the utility of the modules without associated hands-on experience would be limited. Our curriculum utilized the high rate of pathology among patients in the PICU to provide residents with experiential learning and examples of abnormal POCUS findings. It is possible that during the PICU rotation, no patients would provide examples of abnormal findings. In that case, the residents would only have exposure to normal findings and the abnormal examples in the modules. Commercially available or homemade ultrasound simulators can provide examples of abnormal findings if no patients are available but can be expensive or difficult to construct. ${ }^{10}$ Additional ultrasound machines, ideally one for every two residents, would allow for more hands-on ultrasound scanning but are not often feasible given the limited number of ultrasound machines available at most institutions. Future efforts in POCUS education for pediatric residents should include identification of relevant POCUS applications, development of skill assessment tools, and integration of POCUS training across multiple clinical settings to create a longitudinal POCUS curriculum.

Ryan Good, MD: Assistant Professor, Department of Pediatrics, University of Colorado School of Medicine

Jonathan Orsborn, MD: Assistant Professor, Department of Pediatrics, University of Colorado School of Medicine

Timothy Stidham, MD: Assistant Professor, Department of Pediatrics, University of Colorado School of Medicine

\section{Disclosures}

None to report.

\section{Funding/Support}

None to report.

\section{Ethical Approval}

Colorado Multiple Institutional Review Board approved this study.

\section{References}

1. Moore CL, Copel JA. Point-of-care ultrasonography. N Engl J Med. 2011;364(8):749-757. https://doi.org/10.1056/NEJMra0909487

2. Dinh VA, Fu JY, Lu S, Chiem A, Fox JC, Blaivas M. Integration of ultrasound in medical education at United States medical schools: a national survey of directors' experiences. J Ultrasound Med. 2016;35(2):413-419. https://doi.org/10.7863/ultra.15.05073

3. Emergency ultrasound guidelines. Ann Emerg Med. 2009;53(4):550-570. https://doi.org/10.1016/j.annemergmed.2008.12.013

4. Marin JR, Lewiss RE, American Academy of Pediatrics, et al. Point-of-care ultrasonography by pediatric emergency medicine physicians. Pediatrics. 2015;135(4):e1113-e1122. https://doi.org/10.1542/peds.2015-0343

5. Expert Round Table on Ultrasound in ICU. International expert statement on training standards for critical care ultrasonography Intensive Care Med. 2011;37(7):1077-1083. https://doi.org/10.1007/s00134-011-2246-9

6. Lim S-J, Hayden S, Kritek P, et al. Point-of-care ultrasound for critical care. MedEdPORTAL. 2013;9:9619. https://doi.org/10.15766/mep_2374-8265.9619

7. Frost D, Nadjafi M, Cavalcanti R. A curriculum for ultrasound-enhanced physical examination rounds. MedEdPORTAL. 2013;9:9625. https://doi.org/10.15766/mep_2374-8265.9625 


\section{MedEdPORTAL \\ The Journal of Teaching and Learning Resources}

8. Marin JR, Abo AM, Arroyo AC, et al. Pediatric emergency medicine point-of-care ultrasound: summary of the evidence. Crit Ultrasound J. 2016;8:16. https://doi.org/10.1186/s13089-016-0049-5

9. Nguyen J, Amirnovin R, Ramanathan R, Noori S. The state of point-of-care ultrasonography use and training in neonatalperinatal medicine and pediatric critical care medicine fellowship programs. J Perinatol. 2016;36(11):972-976. https://doi.org/10.1038/jp.2016.126

10. Rippey J, Gawthrope I. Creating thoracic phantoms for diagnostic and procedural ultrasound training. Australas J Ultrasound Med. 2012;15(2):43-54. https://doi.org/10.1002/j.2205-0140.2012.tb00226.x

Received: September 5, 2017 | Accepted: February 5, 2018 | Published: February 15, 2018 